\title{
80. Microencapsulation of Mitomycin C for Intra. arterial Infusion Chemotherapy
}

\author{
By Tetsuro Kato and Ryosuke Nemoto \\ Department of Urology, Akita University School of Medicine, Akita \\ (Communicated by Toshio Kurokawa, M. J. A., Sept. 12, 1978)
}

A great deal has been discussed concerning current and future application for encapsulating the materials whose original activities or stabilities may be controlled by microencapsulation, ${ }^{1), 2)}$ but clinical interest in advantages claimed for pharmaceutical microencapsulation is still limited. If anticancer drugs were encased in a microscopic size capsule readily transportable to desired sites where the packaged drugs should be released for prolonged periods of time, an effective regimen of cancer chemotherapy could be facilitated. Since there have been no available anticancer drugs in such a form, we prepared the Mitomycin C-microcapsule (MMC-m.c.) which could be applied to intra-arterial infusion chemotherapy.

Materials and methods. Preparation of MMC-m.c. Principles of coacervation ${ }^{3)}$ are used with certain modifications. A mixture of ethylcellulose $(0.5 \mathrm{~g})$, polyethylene $(0.5 \mathrm{~g})$ and cyclohexane $(50 \mathrm{ml})$ was dissolved in a beaker by heating to $80^{\circ} \mathrm{C}$, and $2 \mathrm{~g}$ of Mitomycin $\mathrm{C}$ (MMC, Kyowa Hakko Co., Ltd.) was dispersed in this solution. The mixture was then gradually cooled to $25^{\circ} \mathrm{C}$ with gentle stirring. In this process the dissolved ethylcellulose encapsulated the particles of MMC. The capsules were rinsed 5 times with n-hexane supplemented with $1 \%$ polyethylene derivative of hydrogenated castor oil (HCO-60, Nikko Chemicals Co.), collected with a 45 mesh screen and air-dried at $45^{\circ} \mathrm{C}$ for $6 \mathrm{hr}$. Thus the capsules were expected to consist of, on average, $80 \%(\mathrm{~W} / \mathrm{W})$ of $\mathrm{MMC}$ as the core and $20 \%(\mathrm{~W} / \mathrm{W})$ of ethylcellulose as the shell coated with a thin film of castor oil.

Surface structure and particle size. MMC-m.c. was scanned with a JEM-U3 scanning electron microscope following vacuum-coating with carbon and gold-palladium $(60: 40)$. The particle size represented by the longitudinal diameter was measured with a micrometer.

Bioassay of MMC-m.c. $50 \mathrm{mg}$ of MMC-m.c. was ground down into powder, mixed well with $50 \mathrm{ml}$ of $0.9 \% \mathrm{NaCl}$ solution (saline) and filtered through a filter paper (No.51A). The biological activity of the filtrates was determined by the agar plate diffusion method ${ }^{4}$ using E. coli B-ATCC 11303 as the target microorganism.

Release rate of $M M C$ from $M M C-m . c .25 \mathrm{mg}$ of MMC-m.c. was 
dispersed in $25 \mathrm{ml}$ of saline at $37^{\circ} \mathrm{C}$, settled at a stationary state or stirred at $5 \mathrm{rpm}$ and filtered as a function of time after the incubation. MMC concentration of the filtrates was assessed spectrophotometrically by measuring the optical density at $360 \mathrm{~m} \mu$ (E\% at $360 \mathrm{~m} \mu=650)^{5} .^{5} 20 \mathrm{mg}$ of non-encapsulated MMC was processed in the same manner for the controls.

Intra-arterial infusion of $M M C-m . c$. A Kifa red catheter (1.2 $\mathrm{mm}$ in internal diameter) was inserted into the left renal artery of mongolian dogs weighing $12-15 \mathrm{~kg}$ through the right femoral artery. The animals were anesthetized with intravenous injection of pentobarbital and the catheterization was aided by laparotomy. MMC-m.c. containing $10 \mathrm{mg}$ of MMC was suspended in $60 \mathrm{ml}$ of saline and infused into the left kidney through the catheter (MMC-m.c. group, 5 dogs). For the controls, $10 \mathrm{mg}$ of non-encapsulated MMC dissolved in $60 \mathrm{ml}$ of saline was infused (control group, $3 \mathrm{dogs}$ ). Infusion time was set for $15 \mathrm{~min}$ in both experimental groups. Blood samples were obtained from the right common iliac vein at the determined intervals. Bilateral nephrectomy was performed $6 \mathrm{hr}$ after the drug infusion and 3 tissue specimens were sampled from the upper, middle and lower transverse sections of each kidney. Bioassay for MMC in serum and kidney tissue homogenate was performed.

Results and discussion. MMC-m.c. formed an irregular particle with a rough surface (Fig. 1). Certain improvements of microencapsulation provided MMC-m.c. with the particle size of $224.6 \pm 54.9 \mu \mathrm{m}$ (mean \pm S.D., $n=100$ ), so that the capsules were readily infused through a Kifa red catheter as well as an 18-gauge needle.

Duplicated bioassay for MMC-m.c. revealed that $50 \mathrm{mg}$ of MMC-

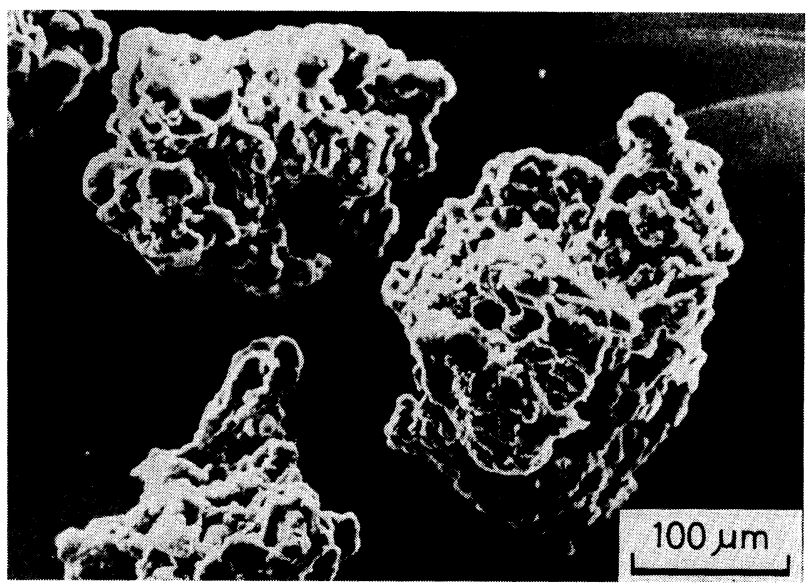

Fig. 1. Scanning electron microscopic finding of MMC-m.c. with particle size of $224.6 \pm 54.9 \mu \mathrm{m}$ (mean \pm S.D., $n=100$ ). 


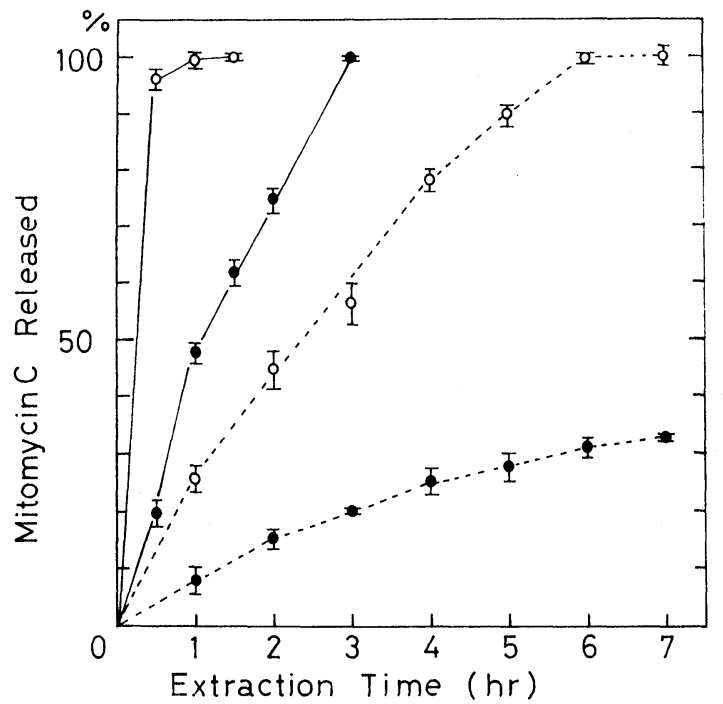

Fig. 2. Release rate of MMC from MMC-m.c. (- $-5 \mathrm{rpm}$, - - - $0 \mathrm{rpm}$ ) and non-encapsulated MMC (-O- $5 \mathrm{rpm}$, -.$--0 \mathrm{rpm})$ in saline at $37^{\circ} \mathrm{C}$; bars, mean $\pm . \mathrm{S} . \mathrm{D}(\mathrm{n}=3)$.

m.c. contained $40.5 \mathrm{mg}(81 \%, \mathrm{~W} / \mathrm{W})$ and $42.0(84 \%, \mathrm{~W} / \mathrm{W})$ of $\mathrm{MMC}$, respectively. This result is in good agreement with the predicted MMC content $(80 \%, \mathrm{~W} / \mathrm{W})$ in the capsule, indicating that the microencapsulating process does not affect the biological activity of MMC.

Sustained release of MMC from MMC-m.c. was clearly demonstrated by the in vitro assay (Fig. 2). MMC was released from the capsules by $31 \pm 1.5 \%$ (mean \pm S.D., $\mathrm{n}=3$ ) at $6 \mathrm{hr}$ stationary incubation, while non-encapsulated MMC was thoroughly dissolved by this time point. MMC was released by $48 \pm 2.3 \%$ at $1 \mathrm{hr}$ rotating incubation, when non-encapsulated MMC was entirely dissolved. It took $3 \mathrm{hr}$ for MMC-m.c. to be completely released into the stirring saline.

The animal experiment showed that MMC levels in circulating blood of the MMC-m.c. group were significantly lower than those of the control group through $30 \mathrm{~min}$ after the intra-arterial infusion $(p<0.01$, Fig. 3). The peak of MMC level, observed 5 min after the drug infusion, was $0.580 \pm 0.159 \mu \mathrm{g} / \mathrm{ml}($ mean \pm S.D., $\mathrm{n}=5$ ) in the MMC-m.c. group, while that in the control group $(n=3)$ was $2.166 \pm$ $0.340 \mu \mathrm{g} / \mathrm{ml}(\mathrm{P}<0.005)$. The peak of MMC level in the MMC-m.c. group can be attributed largely to dissolution of MMC from the capsules during the intra-arterial infusion. In terms of bioavailability, calculated by the area under the blood level curve, the MMC-m.c. group was estimated to release MMC into circulating blood by approximately $40 \%$ relative to the control group. The reduced bio- 


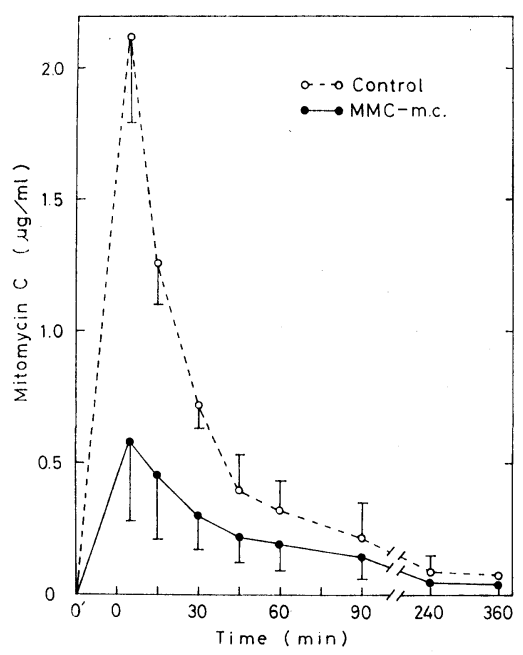

Fig. 3. Blood levels of MMC after intra-arterial infusion with MMC-m.c. containing $10 \mathrm{mg}$ of $\mathrm{MMC}(--\infty)$ and $10 \mathrm{mg}$ of non-encapsulated MMC (-- O --) into dog kidney. $0^{\prime}-0$, infusion time (15:min); bars, mean \pm S.D. $(n=5$ for MMC-m.c., $\mathrm{n}=3$ for control).

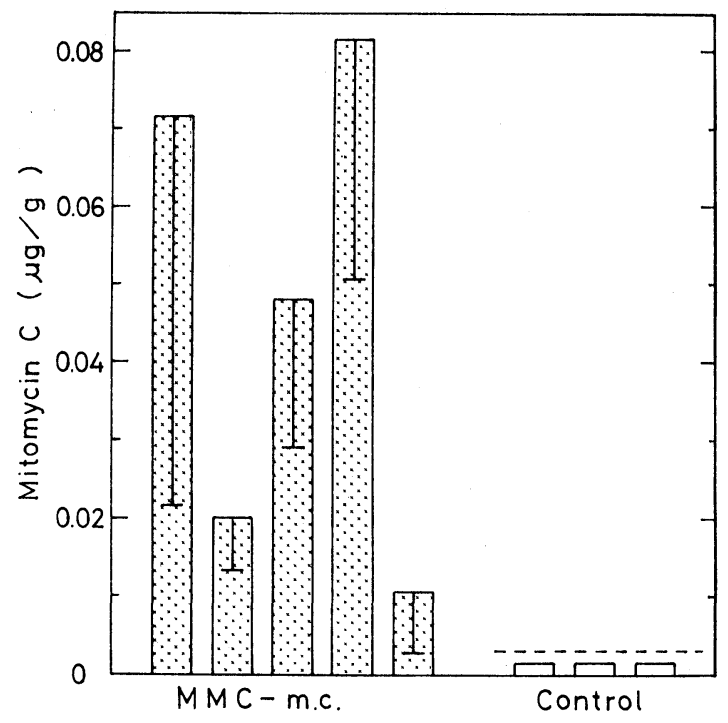

Fig. 4. MMC concentration in dog kidney $6 \mathrm{hr}$ after intra-arterial infussion with MMC-m.c. (5 kidneys) and non-encapsulated MMC (control, 3 kidneys). Bars, mean \pm S.D. $(n=3)$; dotted line, limitation of bioassay.

availability in the MMC-m.c. group is indicative of less toxic effects on non-target tissues. On the other hand, the kidneys of the MMC- 
m.c. group still retained biologically active MMC $(0.046 \pm 0.014 \mu \mathrm{g} / \mathrm{g}$, $\mathrm{n}=15) 6 \mathrm{hr}$ after the arterial infusion, while MMC could not be detected in the control group (Fig. 4). This finding indicates that MMC-m.c. does keep its biological potential for prolonged periods of time even in the kidney which has a potent enzyme system inactivating MMC. ${ }^{5)}$

The present results indicate that microencapsulation of MMC provides a sustained release of biologically active MMC in vitro as well as in vivo and that intra-arterial infusion of the microencapsulated anticancer drugs is applicable to an intensive topical chemotherapy with less systemic toxicity as compared to the non-encapsulated anticancer drugs. Clinical application of MMC-m.c. is currently being investigated by us.

Acknowledgements. We thank Mr. Akira Kondo for technical assistance in microencapsulation and are grateful to Emeritus Professor Toshio Kurokawa, M. J. A., for his critical review of this manuscript.

\section{References}

1) Luzzi, L. A.: Review article. Microencapsulation. J. Pharm. Sci., 59, 13671376 (1970).

2) Nixon, J. R.: Microencapsulation, Marcel Dekker Inc., New York-Basel (1976).

3) Kondo, A.: Microcapsule, Industrial Daily News, Tokyo, pp. $95 \$ 96$ (1970).

4) Miyamura, S.: A determination method for anticancer action of antibiotics by the agar plate diffusion technique. Antibiot. Chemother., 6, 280-282 (1956).

5) Schwartz, H. S., and Philip, E. S.: Pharmacology of mitomycin C. II. Renal excretion and metabolism by tissue homogenates. J. Pharmacol. Exp. Ther., 133, 335-342 (1961). 